\title{
Spontaneous Splenic Rupture as a Paradoxical Reaction during Treatment for Splenic Tuberculosis
}

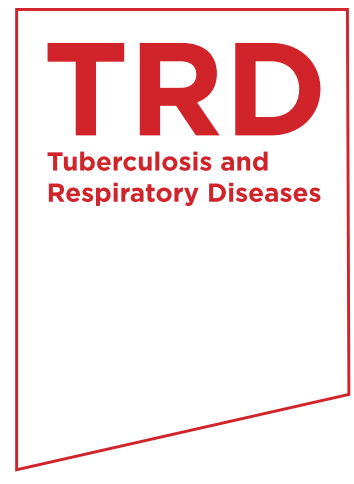

\author{
Hye Ju Yeo, M.D. ${ }^{1}$, Soo Yong Lee, M.D. ${ }^{1}$, Eunyoung Ahn, M.D. ${ }^{1}$, Eun Jung Kim, M.D. ${ }^{1}$, Dae Gon Rhu, \\ M.D. ${ }^{1}$, Kyoung Un Choi, M.D. ${ }^{2}$, Seung Eun Lee, M.D. ${ }^{1}$, Woo Hyun Cho, M.D. ${ }^{1}$, Doosoo Jeon, M.D. ${ }^{1}$ \\ and Yun Seong Kim, M.D. ${ }^{1}$
}

Departments of ${ }^{1}$ Internal Medicine and ${ }^{2}$ Pathology, Pusan National University Yangsan Hospital, Pusan National University

School of Medicine, Yangsan, Korea

This report describes a rare case of a patient with splenic tuberculosis (TB) who developed spontaneous splenic rupture after 10 weeks of antituberculous chemotherapy. The patient responded well to the antituberculous regimen prior to the spontaneous splenic rupture. We considered a paradoxical reaction as a cause of the splenic rupture. The patient underwent splenectomy and continuously received initial antituberculous drugs without change. To the best of our knowledge, this is the first report of spontaneous splenic rupture as a paradoxical reaction to antituberculous chemotherapy in an immunocompetent host with splenic TB.

Keywords: Tuberculosis; Tuberculosis, Splenic; Splenic Rupture; Disease Progression

\section{Introduction}

Splenic tuberculosis (TB) is a rare form of extra-pulmonary $\mathrm{TB}^{1}$. It usually occurs in the disseminated or miliary form of TB, especially in human immunodeficiency virus (HIV)infected patients ${ }^{2}$. Very few studies have reported spontaneous splenic rupture in splenic $\mathrm{TB}^{3-10}$. The majority of these cases occurred in immunocompromised patients ${ }^{3-7}$ or before

Address for correspondence: Doosoo Jeon, M.D.

Department of Internal Medicine, Pusan National University Yangsan Hospital, Pusan National University School of Medicine, 20 Geumo-ro, Yangsan 626-787, Korea

Phone: 82-55-360-1415, Fax: 82-55-360-1757

E-mail: sooli10kr@yahoo.co.kr

Received: May 10, 2012

Revised: Jun. 19, 2012

Accepted: Jul. 19, 2012

(c) It is identical to the Creative Commons Attribution Non-Commercial License (http://creativecommons.org/licenses/by-nc/3.0/).

Copyright (c) 2013

The Korean Academy of Tuberculosis and Respiratory Diseases. All rights reserved. administration of antituberculous chemotherapy ${ }^{3-5,8,9}$. Spontaneous rupture as a paradoxical reaction during successful chemotherapy has not been reported in an immunocompetent patient. We report this case in light of rarity and clinical importance of early recognition for this phenomenon.

\section{Case Report}

A 33-year-old woman (married, homemaker) presented with frequent high-grade fever and left-sided abdominal pain that had lasted for 1 month. There was no history of cough, sputum, or any other respiratory symptoms. She had no history of diabetes, hypertension, TB, or other significant illness. Physical examination showed that her body temperature was $38^{\circ} \mathrm{C}$, pulse rate 105 beats $/ \mathrm{min}$, and blood pressure 100/60 $\mathrm{mm} \mathrm{Hg}$. Abdominal examination revealed an enlarged and tender spleen. Routine examinations of other systems showed unremarkable findings. The patient's laboratory data showed a white blood cell count of $11,590 / \mathrm{mm}^{3}$, hemoglobin concentration $9.4 \mathrm{~g} / \mathrm{dL}$, erythrocyte sedimentation rate $30 \mathrm{~mm} /$ $\mathrm{hr}$, and C-reactive protein concentration $9.02 \mathrm{mg} / \mathrm{dL}$. Other biochemical parameters, including liver functions and levels 

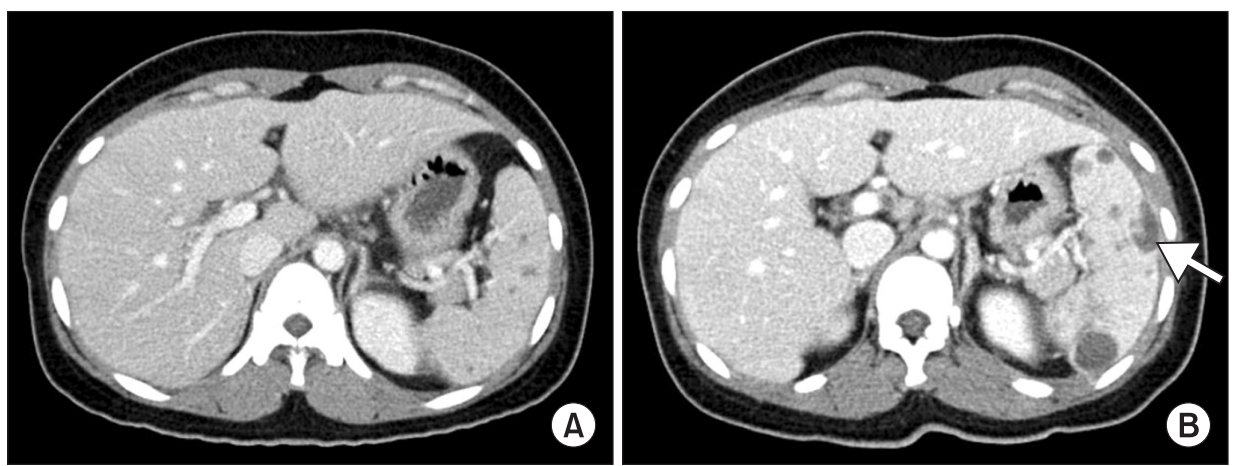

Figure 1. Computed tomography (CT) findings of the abdomen. (A) Initial CT shows multiple hypodense lesions in the spleen. (B) After 10 weeks of chemotherapy, CT shows more increase in the size and number of splenic abscesses with intraperitoneal rupture (arrow).
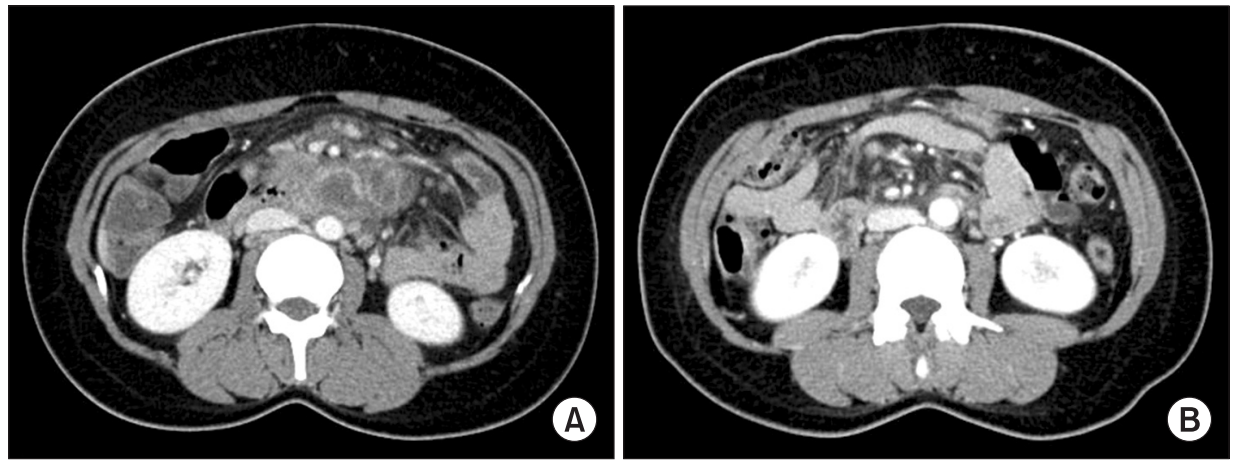

Figure 2. Computed tomography (CT) findings of the abdomen. (A) Initial CT shows several central low density with peripheral rim enhanced lymph nodes enlargement around small bowel mesentery. (B) After 10 weeks of chemotherapy, CT shows decreased interval in the size and number of those lymph node lesions.

of blood sugar, blood urea, and serum creatinine, were within normal limits. T-SPOT.TB assay (Oxford Immunotec, Oxford, UK) showed positive result and enzyme-linked immunosorbent assay showed that the patient was seronegative for antiHIV antibodies. Chest radiography revealed no remarkable abnormalities. Computed tomography (CT) of the abdomen revealed multiple low-density lesions of variable size, with some septations, in the spleen (Figure 1A). We also observed several enlarged mesenteric lymph nodes, which showed central low attenuation with a peripheral rim enhancement, suggestive of TB lymphadenitis (Figure 2A). An ultrasoundguided splenic biopsy revealed inflammatory cells with focal necrosis, and results of acid-fast bacilli (AFB) staining and TB-polymerase chain reaction were negative. Laparoscopic biopsy of the small bowel mesentery was performed for differential diagnosis and to exclude malignancy. However, biopsy of abdominal lymph nodes could not be performed, because profuse bleeding was expected as a result of severe adhesion of small bowel mesentry. Histopathological examination of the biopsy specimen revealed evidence of both acute and chronic inflammation without granuloma. Our initial impression, based on the clinical and radiological findings, was splenic TB accompanied with abdominal TB lymphadenitis. We initiated antituberculous chemotherapy with isoniazid, rifampicin, ethambutol, and pyrazinamide. After 1 week of chemotherapy, the patient became afebrile and did not complain of abdominal pain. She was subsequently discharged.

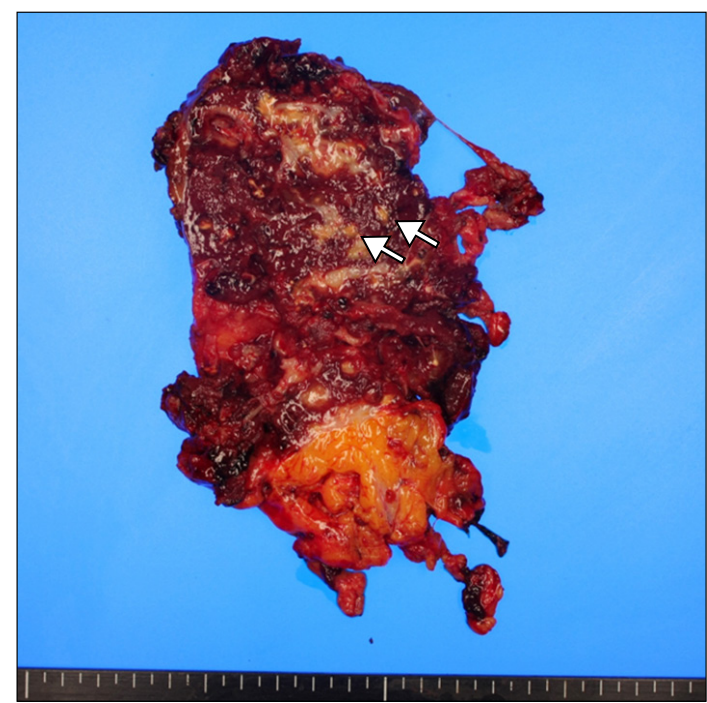

Figure 3. Explanted spleen. By gross appearance, the spleen was enlarged, measuring approximately $14 \times 9 \times 4 \mathrm{~cm}$ in dimension and $232.8 \mathrm{~g}$ in weight. The specimen shows multiple yellow necrotic nodules (arrows) and foci of hemorrhage.

After 10 weeks of chemotherapy, the patient returned to the emergency department of our hospital with sudden-onset dull and aching pain in the abdominal upper left quadrant. Abdominal CT scan showed more increase in the size and 

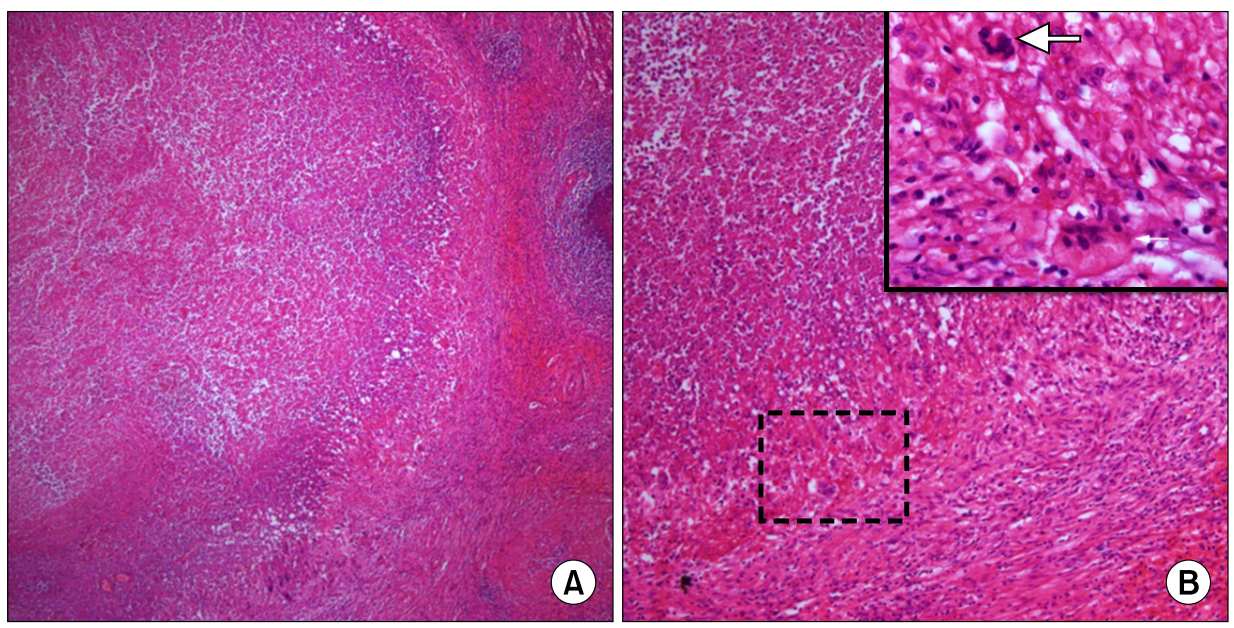

Figure 4. Histopathological findings of the spleen. (A) Large granuloma with central necrosis is noted (H\&E stain, $\times 40)$. (B) Epithelioid and Langhans giant cells (box: arrow) are identified at the peripheral portion of the granuloma (H\&E stain, $\times 100)$.

number of splenic abscesses with intraperitoneal rupture (Figure 1B), whereas more decrease in the size and number of abdominal lymph nodes than were previously observed (Figure 2B). The patient did not report occurrence of recent trauma. The patient was admitted for emergency splenectomy. Gross findings included multiple yellow necrotic nodules and hemorrhagic foci with a lacerated splenic capsule (Figure 3). Histopathological examination showed epithelioid granulomas composed of epithelioid cells, lymphocytes, and Langerhans giant cells with variable degree of central caseous necrosis, consistent with TB (Figure 4). AFB staining and culture for TB from the splenectomy specimen were negative. Considering the clinical improvement of the patient and radiological improvement of concomitant abdominal lymph nodes, we assumed that the splenic rupture was precipitated by paradoxical reaction. Antituberculous chemotherapy was continued postoperatively without regimen change, for a total treatment time of 9 months. The patient is currently clinically disease-free for 3 months after completion of chemotherapy.

\section{Discussion}

Splenic TB is an extremely rare cause of spontaneous splenic rupture ${ }^{11,12}$. Nearly $90 \%$ of splenic ruptures are caused by trauma. The remaining $10 \%$ are due to underlying diseases, such as splenic angiomas, infarctions, neoplasms, and infectious diseases ${ }^{11}$.

A few cases of spontaneous rupture of splenic TB have been reported previously in a single case report ${ }^{3-10}$. The majority of these cases occurred in immunocompromised patients. These patients had HIV infection ${ }^{3,4,7}$, longstanding sarcoidosis ${ }^{5}$, or chronic renal disease requiring hemodialysis ${ }^{6}$. Thus far, 3 cases have been reported in immunocompetent patients $^{8-10}$. Splenic hemangioma was a factor in one of these cases $^{8}$.

Most spontaneous ruptures in splenic TB were reported as initial manifestations, occurring before initiation of antituberculous chemotherapy ${ }^{3-5,8,9}$. This suggests that the diagnosis of splenic TB is often delayed due to the lack of specific symptoms and signs. Three cases of spontaneous splenic rupture have been reported during antituberculous chemotherapy. The first case occurred 1 week after initiation of chemotherapy in an immunocompetent patient $t^{10}$. The second case occurred as a result of treatment failure in a patient with longstanding sarcoidosis ${ }^{5}$. The third case occurred after 4 weeks of chemotherapy as a manifestation of immune reconstitution inflammatory syndrome in an HIV-infected patient ${ }^{7}$. Spontaneous splenic rupture occurring as a paradoxical reaction during successful chemotherapy has not been reported in an immunocompetent patient.

In the present case, splenic rupture occurred after 10 weeks of chemotherapy. Before splenic rupture, the patient's condition was improving. The initial symptoms of fever and abdominal pain had resolved. In addition, abdominal CT performed at the time of splenic rupture showed improvement of lymph node lesions. Postoperatively, the patient was continuously treated with initial antituberculous drugs and successfully completed treatment. Therefore, we consider paradoxical reaction as the cause of spontaneous splenic rupture.

A paradoxical reaction is defined as radiological and clinical worsening of a previous lesion or the development of new lesions after initial improvement during antituberculous treatment ${ }^{13,14}$. This has been observed in cases of intracranial tuberculoma, TB lymphadenitis, and pleural and pulmonary $\mathrm{TB}^{13,14}$

The pathogenesis of paradoxical reaction is generally regarded as the hypersensitivity response to mycobacterial antigens rather than high bacillary load. This immunological hypothesis is supported by a relatively low mycobacterial culture rate and significant response after the use of corticosteroids ${ }^{13,14}$. In our case, AFB staining and culture for TB from the splenectomy specimen were negative. We presumed that negative microbiological result related with the paradoxical 
reaction as well as 10 weeks of antituberculous theapy prior to the splenectomy.

Paradoxical reactions in TB may be localized or systemic phenomena. Different responses of multiple lesions to antituberculous therapy were reported as a result of paradoxical reaction ${ }^{15}$. Our case showed different response of spleen and abdominal lymph nodes to antituberculous treatment. By contrast to the splenic lesions, we could not obtain microbiological or histological evidences for TB in abdominal lymph node lesions. However, the CT scan demonstrated typical radiological findings suggestive of TB lymphadenitis and improvement of those lesions during treatment. Therefore, presumptive diagnosis for abdominal TB lymphadenitis could be allowed based on these findings. In addition, different responses of spleen and abdominal lymph nodes might be one of the findings to support the paradoxical reaction as a cause of splenic rupture.

It is not clear how paradoxical reaction results in splenic rupture. We propose that subcapsular localization of granulomatous TB lesions causes tearing of the splenic capsule. Rapid mycobacterial killing following successful chemotherapy may release mycobacterial products, which stimulate production of cytokines. These cytokines then enhance focal immune responses with accumulation of inflammatory exudates, ultimately resulting in spleen rupture.

Despite the rarity of this condition, this case has clinical importance because it encourages awareness for this phenomenon. It is clinically important to distinguish paradoxical reactions from treatment failures because paradoxical responses usually do not require changes in therapy. Even occult rupture is associated with high mortality if unrecognized; therefore, spontaneous rupture of the spleen usually requires surgical intervention. All but one ${ }^{7}$ reported cases of splenic rupture in splenic TB have been treated with splenectomy.

In summary, we described a case of spontaneous splenic rupture as a paradoxical reaction in an immunocompetent patient with splenic TB. The patient was successfully treated with splenectomy and continuing antituberculous chemotherapy with initial drugs. To the best of our knowledge, this is the first case report of splenic rupture during successful antituberculous therapy in an immunocompetent host.

\section{References}

1. Peto HM, Pratt RH, Harrington TA, LoBue PA, Armstrong LR. Epidemiology of extrapulmonary tuberculosis in the United States, 1993-2006. Clin Infect Dis 2009;49:1350-7.
2. Sharma SK, Smith-Rohrberg D, Tahir M, Mohan A, Seith A. Radiological manifestations of splenic tuberculosis: a 23-patient case series from India. Indian J Med Res 2007;125:66978.

3. Pramesh CS, Tamhankar AP, Rege SA, Shah SR. Splenic tuberculosis and HIV-1 infection. Lancet 2002;359:353.

4. Rathore S, George P, Deodhar M, Calton N, George U, Pawar $\mathrm{B}$, et al. Spontaneous rupture of tuberculous spleen in a HIV seropositive patient on maintenance hemodialysis. Saudi J Kidney Dis Transpl 2009;20:822-5.

5. Lam KY, Ng WF, Chan AC. Miliary tuberculosis with splenic rupture: a fatal case with hemophagocytic syndrome and possible association with long standing sarcoidosis. Pathology 1994;26:493-6.

6. Ram R, Swarnalatha G, Prasad N, Murty KV. Pathological rupture of spleen in a haemodialysis patient due to tuberculosis. Nephrol Dial Transplant 2006;21:2994-5.

7. Weber E, Gunthard HF, Schertler T, Seebach JD. Spontaneous splenic rupture as manifestation of the immune reconstitution inflammatory syndrome in an HIV type 1 infected patient with tuberculosis. Infection 2009;37:163-5.

8. Rao RC, Ghose R, Sawhney S, Berry M. Hemangioma of spleen with spontaneous, extra-peritoneal rupture, with associated splenic tuberculosis: an unusual presentation. Australas Radiol 1993;37:100-1.

9. Safioleas MC, Stamatakos MC, Safioleas CM, Diab AI, Agapitos EB. Co-existence of spontaneous splenic rupture and tuberculosis of the spleen. Saudi Med J 2006;27:1588-90.

10. Fegan D, Butcher C, Rees C, Glennon J. Tuberculosis and abdominal pain. Intern Med J 2005;35:437-8.

11. Sterlacci W, Heiss S, Augustin F, Tzankov A. Splenic rupture, beyond and behind: a histological, morphometric and followup study of 254 cases. Pathobiology 2006;73:280-7.

12. Renzulli P, Hostettler A, Schoepfer AM, Gloor B, Candinas D. Systematic review of atraumatic splenic rupture. Br J Surg 2009;96:1114-21.

13. Breen RA, Smith CJ, Bettinson H, Dart S, Bannister B, Johnson MA, et al. Paradoxical reactions during tuberculosis treatment in patients with and without HIV co-infection. Thorax 2004;59:704-7.

14. Carvalho AC, De Iaco G, Saleri N, Pini A, Capone S, Manfrin M, et al. Paradoxical reaction during tuberculosis treatment in HIV-seronegative patients. Clin Infect Dis 2006;42:893-5.

15. Park KY, Lee HJ, Jung JW, Choi YH, Nam SB, Ahn SH, et al. A case of different response of miliary lung and intracranial nodules to antituberculous therapy. Tuberc Respir Dis 2008;64:153-7. 